\title{
2013 Conant Prize
}

JOHN BAEZ and JOHN HUERTA received the 2013 Levi L. Conant Prize at the 119th Annual Meeting of the AMS in San Diego, California, in January 2013.

\section{Citation}

The 2013 Levi L. Conant Prize is awarded to John Baez and John Huerta for their article, "The algebra

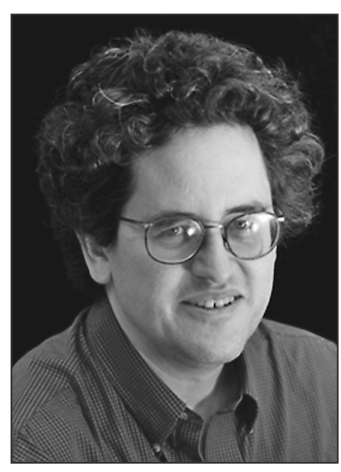
of grand unified theories", Bulletin of the American Mathematical Society 47 (2010), no. 3, 483-552.

The Standard Model of particle physics is one of the central theoretical constructs of twentieth-century physics. It attempts to describe all particles and all the forces of nature except gravity. Although the Standard Model seems complicated and somewhat arbitrary, it has been very successful in describing mathematically what we see in reality. Physicists are not fully satisfied with it because it does leave out

John Baez gravity, described by Einstein's general

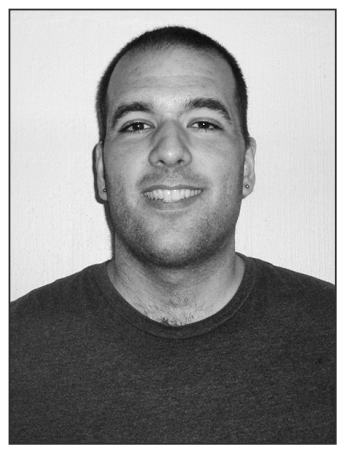
theory of relativity, which has not been reconciled with the Standard Model, and it may not be able to account for dark matter. Looking beyond the Standard Model leads to string theory, loop quantum gravity, and theories based on noncommutative geometry.

In the second half of the twentieth century, a program of "grand unification" commenced whose aim was to unify particles and the forces, save gravity, within the confines John Huerta of the Standard Model using Lie groups, Lie algebras, and their representations. Such a theory features a compact Lie group $G$, called the gauge group. Particles lie in a representation of $G$ on a finite-dimensional com-

DOI: http://dx.doi.org/10.1090/noti967 plex vector space $V$. More precisely, particles are the basis vectors of the associated irreducible representations on $V$. Ideas coming from the era of grand unification persist into the twenty-first century and are still important today. The article focuses upon the group representation theory that describes particles in the Standard Model. The authors eschew symmetry breaking (particle interaction) so they can limit the discussion to algebraic ideas that mathematicians not coming from theoretical physics will find familiar. Their goal is to introduce the unfamiliar ways in which physicists use these algebraic ideas.

Even with the simplifications inherent in the restricted range of the Standard Model alluded to above, it is a daunting task to convey decades of work in one relatively short article. A grand unified theory is based on different versions of extending the Standard Model. The gauge group of the Standard Model is $G_{S} M=U(1) \times S U(2) \times S U(3)$. An extension of this gauge group, together with an appropriate representation, composes a grand unified theory. The article deals with three such extensions whose gauge groups are $S U(2) \times S U(2)$ $\times S U(4), S U(5)$ and $\operatorname{Spin}(10)$. It transpires that the third theory can be viewed as an extension of the first two, thereby uniting the points of view. The article finishes with some intriguing theorems that lead to interesting speculations about the nature of matter and force in the universe. Along the way, we are treated to a running account of the Standard Model, how it began with the Heisenberg model of the proton and neutron, and how it has evolved with grand unification. Such wonderful physical notions as color, spin, handedness, antimatter, and the like make their appearance along the way. At the same time, concrete theory of the aforementioned Lie groups and their representations are related to these physical notions, and the group theory is shown to lead to interesting 
physical conclusions and puzzles. The authors do an excellent job of keeping the mathematics within reach of even a well-educated, advanced graduate student and alternate nicely between the physics and the mathematics.

The article is a very well-written piece about an interesting and important topic. Too often the expository aspect of an article is limited to the initial sections, so that once a reader is "hooked", he or she has to continue through much drier material. That is not the case in this article. The authors are able to maintain an engaging tone throughout the seventy pages it takes to tell this marvelous tale. And as with the stories in the Thousand and One Nights, we are left hoping for more.

\section{Biographical Sketches}

John Baez is professor of mathematics at the University of California Riverside. Until recently he worked on higher category theory and quantum gravity. His Internet column "This Week's Finds" dates back to 1993 and is sometimes called the world's first blog. In 2010, concerned about climate change and the future of the planet, he switched to working on more practical topics and started the Azimuth Project, an international collaboration to create a focal point for scientists and engineers interested in saving the planet.

John Huerta is starting a postdoctoral fellowship at Instituto Superior Técnico in Lisbon, where he will work with Roger Picken on higher gauge theory and its role in string theory. Before that he was a postdoc at the Australian National University, where he studied the smallest exceptional Lie group and its relationship with a rolling ball. He was a Ph.D. student of John Baez, studying supersymmetry, higher gauge theory, and their connection to division algebras. Though he studied mathematics, he's always loved physics and derives great inspiration from it. His interest began with a popular astronomy book that his sister gave to him as a child. This evolved into a fascination with stars, with physical laws, and eventually the underlying mathematics and its conceptual interplay.

\section{Responses}

From John Baez: I put a lot of energy into explaining math and physics online. Blogging is no substitute for more formal writing about academic subjects, but it fills a gap, especially for the millions who don't live near a good research university. Socrates complained that "writing is unfortunately like painting, for the creations of the painter have the attitude of life, yet if you ask them a question they preserve a solemn silence." This is no longer true with blogs: the author is there to answer your questions! So, I am hoping that eventually blogs will be taken seriously by academia, and the AMS will have an award for the best mathematics blog. But I am very happy to receive this prize for a more traditional form of mathematics exposition.

From John Huerta: It is a great honor to receive this award and to share it with my mentor, John Baez. I know there were many other worthy contenders for this award, which only adds to my gratitude. I hope, if nothing else, the attention this honor brings leads more people to learn this wonderful story we did our best to tell. This was our first paper together, and my first publication. I remain deeply proud of it, but it belongs to more people than I can name. I'd like to thank the AMS for their top-notch editors, UC Riverside Mathematics for having me as a student, my parents and my friends for their love and support. But most of all, I want to thank John, for being tough and for being kind, and for teaching me how to write.

\section{About the Prize}

The Conant Prize is awarded annually to recognize an outstanding expository paper published in either the Notices of the AMS or the Bulletin of the $A M S$ in the preceding five years. Established in 2001, the prize honors the memory of Levi L. Conant (1857-1916), who was a mathematician at Worcester Polytechnic Institute. The prize carries a cash award of US\$1,000.

The Conant Prize is awarded by the AMS Council acting on the recommendation of a selection committee. For the 2013 prize, the members of the selection committee were Thomas F. Banchoff, Jerry L. Bona, and J. Brian Conrey.

Previous recipients of the Conant Prize are: Carl Pomerance (2001); Elliott Lieb and Jakob Yngvason (2002); Nicholas Katz and Peter Sarnak (2003); Noam D. Elkies (2004); Allen Knutson and Terence Tao (2005); Ronald M. Solomon (2006); Jeffrey Weeks (2007); J. Brian Conrey, Shlomo Hoory, Nathan Linial, and Avi Wigderson (2008); John W. Morgan (2009); Bryna Kra (2010); David Vogan (2011); and Persi Diaconis (2012).

-Elaine Kehoe 